\title{
Consumer Adoption of Artificial Intelligence: A Review of Theories and Antecedents
}

\author{
Ransome Bawack \\ ICN Business School, France \\ ransome.bawack@icn-artem.com
}

\author{
Kathleen Desveaud \\ Kedge Business School, France \\ kathleen.desveaud@kedgebs.com
}

\begin{abstract}
Recently, people are increasingly adopting technologies powered by artificial intelligence (AI) in their everyday lives. Several researchers have investigated this phenomenon using several theoretical perspectives to explain the motivations behind such behaviour. Our paper reviews this body of knowledge to highlight the technologies, theories, and antecedents of AI adoption investigated this far in academic research. By analysing publications found in Harzing's Journal Quality List, this paper identifies 52 publications on user adoption of AI, 198 antecedents, and 36 theoretical perspectives used to explain user adoption of AI. The most widely used theoretical perspectives in this area of research are the technology acceptance model (TAM) and the unified theory of acceptance and use of technology (UTAUT). Meanwhile, perceived usefulness, perceived ease of use, and trust are the most studied antecedents. Finally, we discuss the implications of these findings for future research on AI adoption by consumers.
\end{abstract}

\section{Introduction}

The ubiquitous presence of artificial intelligence (AI) technologies like voice assistants, autonomous vehicles, and service robots make their adoption increasingly interesting for information systems (IS) and marketing researchers. Based on existing definitions [1], [2], this paper uses the term AI to describe any technology artefact that can perceive changes in its environment, make sense of it, act on this information, interact with other entities within, and adapt to new environments or situations through learning. Meanwhile, AI adoption refers to users' willingness to accept $\mathrm{AI}$ devices or service use [3].

Recently, many researchers have investigated the adoption of AI-powered services and technologies by consumers. Consumers are individuals that seek to fulfil their needs, wants, desires, or preferences by searching, selecting, acquiring (usually by purchasing), and evaluating products, services, ideas, or experiences [4], [5]. Several studies have focused on financial [6], healthcare [7], transportation [8], and hospitality services [9]. The studies are motivated by the efficiency, productivity, and convenience AI systems provide consumers [10]. Most of the extant research has used well-established theoretical perspectives to explain these antecedents as they would for any non-AI technology. Although these perspectives significantly explain AI adoption by consumers from a technological standpoint, very few studies propose theoretical perspectives and antecedents specific to AI-powered technologies.

Given that with AI comes new challenges, it is a critical gap, and AI acceptance can no longer be explained by traditional theories exclusively. AI technologies evolve in real-time and introduce new challenges, ethical issues, and attributes to the digital ecosystem that non-AI technologies do not possess. Thus, using traditional technology acceptance theories and models would not sufficiently explain consumer adoption of AI [11]. This challenge has led to several calls for research on new theoretical developments and perspectives that focus on the specificities of AI technologies to understand their adoption by consumers [10], [12], [13].

Nevertheless, it is challenging for researchers to propose new theories and antecedents of AI adoption without a clear picture of the current state of research on the topic. A recent publication attempts to fill this research gap and compares three theoretical perspectives and related antecedents [14]. Their proposition is a step towards knowledge integration, but it could be further developed for two main reasons. First, the theoretical frameworks aggregated are rather classic frameworks of technology adoption, and they are not necessarily the most successful in explaining AI adoption. AI is profoundly different from traditional technologies by its human-likeness, which implies acquiring human skills [15]. Secondly, a pre-analysis of 
the existing literature allowed us to identify many theories and concepts used to explain AI adoption that were not covered in previous review studies. Thus, our paper aims to provide a more holistic view of the extant research on AI adoption by consumers. It attempts to answer the research question: what factors and theoretical perspectives have helped explain consumer adoption of AI to date? Based on Harzing's Journal Quality List (JQL) [16], this paper identifies theoretical perspectives and antecedents used to explain AI adoption by consumers published in well-established and internationally recognised peer-reviewed journals. In doing so, this paper contributes to AI adoption research by synthesising the extant body of knowledge and revealing theoretical underpinnings that currently dominate this research area. Despite the vast research interest on the topic, this is the first attempt to provide a holistic view as it brings together disparate streams of research to shed light on this fast-growing phenomenon. Synthesising research on AI adoption by consumers also gives IS and marketing professionals a clearer picture of factors that affect user adoption of AI-powered devices and services.

The following section presents the methodology used to identify relevant papers for this literature review.

\section{Methodology}

We used the approach proposed by Webster and Watson [17] to identify relevant research on the antecedents of AI adoption by consumers and related theoretical perspectives. The approach involves three main steps: (i) search academic databases for relevant literature; (ii) identify relevant articles from the list of references of articles selected in step 1; (iii) identify relevant articles from the list of articles citing articles in steps 1 and 2 .

To ensure the identification of high-quality articles, we restricted our literature search to journals listed in Harzing's JQL [16]. This list consolidates wellestablished peer-review journals listed in internationally-recognised journal quality guides like those provided by the Association of Business Schools (ABS), Australian Business Deans Council (ABDC), and the French National Centre for Scientific Research (CNRS). It contains all the main peer-reviewed journals in business and management fields like IS, marketing, knowledge management, operations research, management science, production \& operations management, economics, finance, and psychology.

To query the selected journals for relevant articles, we developed a search string by conducting a preliminary search in the Web of Science (WoS) core collection of databases using the terms "artificial intelligence" and "adoption". This step enabled us to identify keywords related to AI and adoption. The keywords identified were therefore used to develop a final search string used to search the targeted journals. Relevant articles were identified by searching each JQLlisted journal's title, abstract, and keywords using the following search string: ("artificial intelligence" OR "voice assistant" OR "AI-powered avatar" OR "intelligent personal assistant" OR "personal intelligent agent" OR "robot" OR "chatbot" OR "smart wearable device" OR "automated vehicle") AND ("acceptance" OR "acceptance intention" OR "adoption" OR "adoption intention" OR "use" OR "intention to use" OR "continuance" OR "continuance usage" OR "continuance usage intention" OR "continuous use" OR "reuse" OR "intention to reuse"). To ensure that no relevant article was missed, we searched two additional databases: Web of Science and Business Source Complete. Steps two and three did not lead to any previously unidentified publications. Conducted in April 2021, this search identified 893 articles. Only articles that investigated antecedents of AI adoption by consumers were retained as relevant. Both authors analysed the content of these articles in May 2021 and identified 52 relevant articles.

The following section discusses the findings of this paper.

\section{Results and discussion}

\subsection{Measuring AI adoption}

Figure 1 presents the most frequent terms used to describe adoption in AI adoption literature. Observe that researchers use terms like behavioural intention, intention to use, acceptance, and adoption intention interchangeably to describe AI adoption.

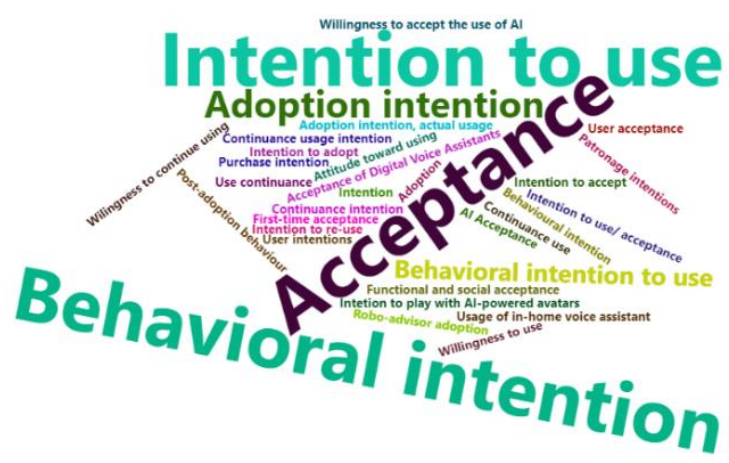

Figure 1. Terms used to describe adoption

They do not often explain what any of these terms mean in the context of their studies[18]-[20]. They 
probably assume that these terms are easily understood and well-known to researchers, but not necessarily the case. This lack of explanation is confusing because researchers often measure behavioural intention to use AI to address AI adoption [21] or AI acceptance [19]. Does this mean AI adoption and acceptance mean the same thing? Conceptually, these terms may refer to different things and may influence the way the concept is measured. For example, acceptance has been conceptualised as a user's demonstrated willingness to use technology for a task it is designed to accomplish [22]. Another study conceptualised user acceptance as a multifaceted concept that describes a user's attitude towards using a system [23]. The study operationalised this concept by assessing direct attitude towards the system. User acceptance has also been described as a positive experience obtained using technology (robots) [24]. The study operationalises user acceptance as functional and social acceptance. User acceptance has also been operationalised through intention to use or willingness to pay for a technology [23].

Meanwhile, adoption has been conceptualised as users' willingness to use a technology (AI device) [3]. Adoption intention has also been conceptualised as the probability that an individual will engage in a specific behaviour (accept a new technology - AI) [9]. This concept has been operationalised using intention to use and users' willingness to buy, just as has been done in AI acceptance studies [8]. Therefore, it is not clear whether in investigating AI, AI adoption and acceptance should mean the same thing and should always be measured the same way. However, the literature highlights that adoption and acceptance are concepts investigated using constructs baring terms like behavioural intention and adoption intention. Thus, it is essential to define what each term means to avoid ambiguity and misinterpretation of concepts.

Definitions from Oxford's English dictionaries could be a great starting point towards understanding these concepts. They define adoption as the action or fact of choosing to take up, follow, or use something; acceptance as the action of consenting to receive or undertake something offered; use as the action of using something or the state of being used for a purpose; and continuance as the state of remaining in existence or operation. Thus, from a grammatical perspective: adoption should focus on the action or fact of choosing to take up, follow, or use AI technology (in this context); acceptance should focus on the act of consenting to receive or undertake an $\mathrm{AI}$ technology offered; use should focus on the action or the state of using AI technology for a purpose; continuance should focus on the state of keeping AI technology use in existence or operation. Take the case of (AI-powered) voice assistants, for example. Following the above logic, an adoption study would investigate whether consumers choose to take up or use voice assistants to perform online activities. If a researcher decides to examine whether consumers would consent to use voice assistants offered by a brand to perform shopping activities, that would be an acceptance study. The difference is that the consumer initiates adoption, while the product/service provider initiates acceptance. If the researcher focuses on the action or state of using voice assistants for online shopping by consumers, then s/he would be conducting a use study. Finally, if a researcher investigates consumer behaviour regarding the continuous use of voice assistants for online shopping observed over time (e.g., six months), that would be a continuance study.

\subsection{AI technologies}

Figure 2 presents the main categories of technologies empirically investigated in AI adoption research and the number of articles found in each category.

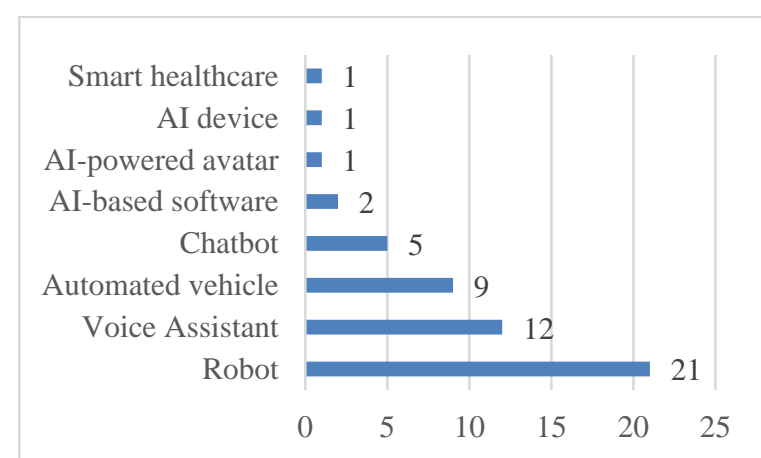

Figure 2. Number of articles found in each category of AI technologies investigated in AI adoption research

Researchers have primarily focused on people's adoption of AI-powered robots (21 articles). For example, some have investigated the adoption of service robots in the tourism and hospitality industry [19], [25][28]. Others have explored the acceptance of news articles written by robot journalists [29]. Others have researched the adoption of social robots in general robots that understand human language and communicate with humans as other humans do in social life [24], [30]-[36]. Meanwhile, some have been more specific about social robot adoption in contexts like retirement homes [37].

The next most researched technology category is voice assistants (12 articles). This category contains articles that focus on the adoption of AI-powered voice assistants like Amazon's Alexa, Google's Google Assistant, and Apple's Siri. Such technologies are 
sometimes referred to as intelligent personal assistants [38], digital voice assistants [39], personal intelligent agents [40], and voice-based assistants [41]. In this category, researchers have investigated AI adoption in general from different theoretical perspectives [38], [40]-[45] (theoretical perspectives will be discussed detailly in the following subsection). Others have focused on the adoption of voice assistants in service delivery [39] and consumer brand management [46].

Autonomous vehicles carry the third-largest number of research papers ( 9 articles). This category groups articles investigating user adoption of AIpowered autonomous vehicles sometimes called selfdriving vehicles [47]. Many researchers have sought to identify general factors that affect user adoption of autonomous vehicles [47]-[49]. Meanwhile, some have focused on cross-cultural differences [23] and the role of trust in user adoption of the technology [50], [51]. Finally, some researchers have also focused on the adoption of autonomous vehicles in the specific case of China [8], [52].

Chatbot is the next main category of consumer AI technologies currently researched (5 articles). The category groups all papers investigating the adoption of AI-powered technologies that emulate dialogue with humans using natural language text. These technologies are trendy nowadays and found on the website of most service companies. Some papers identified in this review investigated consumer adoption of chatbots in the hospitality and tourism industry [9] and Chinese online travel agencies [53]. Others investigated its adoption in customer services [54] and advertising contexts [55].

We found only two studies investigating AI-based software adoption in general, describing software that use AI technologies. One study investigates its adoption in social media marketing [56] and the other in ecommerce systems [57]. Only one study investigated user adoption of AI-powered avatars its adoption in gaming contexts [21]. Also, one paper was found on user adoption of an AI-powered device (smart speaker) [58] and smart healthcare (healthcare services based on AI devices) [7].

\subsection{Theoretical perspectives}

Table 1 presents the theoretical perspectives used in the articles reviewed to explain consumer adoption of AI.

Table 1. Theoretical perspectives used to explain user adoption of AI

\begin{tabular}{|l|l|}
\hline Theoretical perspective & Freq \\
\hline Technology acceptance model (TAM) & 14 \\
\hline
\end{tabular}

\begin{tabular}{|l|l|}
\hline $\begin{array}{l}\text { Unified theory of acceptance and use of } \\
\text { technology (UTAUT) }\end{array}$ & 7 \\
\hline Parasocial relationship theory & 4 \\
\hline Anthropomorphism & 3 \\
\hline Theory of planned behaviour & 3 \\
\hline Flow theory & 2 \\
\hline Innovation diffusion theory & 2 \\
\hline Social distance theory & 2 \\
\hline Uncanny valley & 2 \\
\hline Uncertainty reduction theory & 2 \\
\hline Attribution theory & 1 \\
\hline Behavioural reasoning theory (BRT) & 1 \\
\hline Computers are Social Actors (CASA) paradigm & 1 \\
\hline Consumer acceptance of technology model & 1 \\
\hline Domestication theory & 1 \\
\hline Expectation-confirmation model (ECM) & 1 \\
\hline Family systems theory & 1 \\
\hline Fashion theory & 1 \\
\hline Gender stereotypes & 1 \\
\hline $\begin{array}{l}\text { Lazarus's cognition-motivation-emotion } \\
\text { framework }\end{array}$ & 1 \\
\hline Mind perception theory & 1 \\
\hline Mobile technology acceptance model & 1 \\
\hline Psychological ownership theory & 1 \\
\hline Realism maximisation theory & 1 \\
\hline Ripple effect theory & 1 \\
\hline Service robot acceptance model (sRAM) & 1 \\
\hline Social identity theory & 1 \\
\hline Social learning theory & 1 \\
\hline Social presence theory & 1 \\
\hline Social response theory & 1 \\
\hline Technology readiness theory & 1 \\
\hline The echo effect & 1 \\
\hline Theory of reasoned action & 1 \\
\hline Theory of self-efficacy & 1 \\
\hline Trust in technology model & 1 \\
\hline Uses and gratification theory & 1 \\
\hline & \\
\hline
\end{tabular}

The table presents 36 different theoretical perspectives used by researchers to explain user adoption of AI technologies. These perspectives can be split into two broad categories: classic technology adoption theories and anthropomorphism-oriented theories.

Among the classic technology acceptance and adoption theories, the most widely used theoretical perspective is the technology acceptance model (TAM) proposed by Davis [59] (14 articles). This theoretical model highlights perceived usefulness and perceived ease of use as essential predictors of user acceptance of computer systems. Thus, researchers have used the model to predict the adoption of AI systems like autonomous vehicles [48], [52], chatbots [9], and service robots [26]. Most studies found that perceived 
usefulness and perceived ease of use are strong predictors of behavioural intention to use AI [9], [26], [48], [52]. However, a field experiment in the context of self-driving cars shows that perceived ease of use has no significant effect on continuance use (willingness to reride in a self-driving car) [49].

The unified theory of acceptance and use of technology (UTAUT) [60] is the second most widely used theoretical model in explaining AI adoption by consumers. The theoretical model highlights performance expectancy, effort expectancy, social influence, and facilitating conditions as essential predictors of technology acceptance and use. Thus far, the extant literature shows that performance expectancy, effort expectancy, and social influence significantly affect behavioural intention to use AI [34]. Nevertheless, no study was found that tested the effect of facilitating conditions on AI adoption. Furthermore, the original UTAUT was designed for organisational level assessments. However, an extended version of the model (UTAUT2) was proposed to improve the ability of the model to predict consumer acceptance and use of information technology (IT) [61]. UTAUT2 extends UTAUT by adding hedonic motivation, price value, and habit as critical determinants of consumer acceptance and use of IT. This review identified no study that empirically tested the effect of these variables on consumer adoption of AI technologies.

Researchers have also used the theory of planned behaviour [62] to explain consumer adoption of AI technologies. The theory highlights attitude, subjective norm, and perceived behavioural control as key determinants of behavioural intention and the actual behaviour manifestations. Some researchers found that all three factors influence behavioural intention to use social robots [35], [63]. Meanwhile, others found that attitude and subjective norms affect intention to use but not perceived behavioural control [36]. Other wellknown theories in technology adoption literature have been less often explored to understand the specific context of AI adoption. For example, very few studies have examined AI adoption using theories like the innovation of diffusion theory [64], uncertainty reduction theory [51], expectation-confirmation model [53], and uses \& gratification theory [65]. A study used attribution theory to show that customers' attributions of service enhancements positively affect the intention to use and recommend service robots.

In contrast, attribution of cost reduction negatively affects intention to use service robots but have no significant effect on the intention to recommend the robot [19]. Another study used the behavioural reasoning theory to show that attitude towards AI (autonomous vehicles) and the need for uniqueness positively affects consumers' intention to use AI. In contrast, risk aversion negatively affects those intentions [8]. Trust in technology model and psychological ownership theory were used to show that psychological ownership and perceived trustworthiness positively affect post-adoption behaviour of consumers in terms of cognitive absorption and intention to explore consumer robots [31]. Researchers have also used selfefficacy theory to show the importance of robot use selfefficacy on user acceptance of (care) robots [24]. Nevertheless, this paper also highlights the absence of studies that test some classic technology acceptance theories in the AI context, like Roger's diffusion of innovation theory [66].

The second broad category of theories used by researchers to investigate consumer adoption of $\mathrm{AI}$ is anthropomorphism-oriented. Anthropomorphism giving human attributes to non-human entities, is another increasingly popular theoretical perspective for assessing consumer adoption of AI technologies [67]. This group of theories attempt to explain how humanlike attributes like intelligence, appearance, and social behaviour affect consumer adoption of AI. For example, anthropomorphism has been shown to drive consumer trust, enjoyment, and intention to use service robots [54], [68]. This phenomenon has been explained using the realism maximisation theory [43].

The parasocial relationship theory [69] has also been used to explain consumer adoption of AI. The theory argues that a one-sided perception of intimacy can be developed by individuals vis-a-vis a media personality or any entity that projects human attributes. Based on this logic, some researchers show that parasocial relationships and task attraction lead to satisfaction with AI devices and continuance intention [38]. Combined with the echo effect, ripple effect theory, social learning theory, and family systems theory, the parasocial relationship theory has also helped explain the positive impact of hedonic motivation, compatibility, and perceived security on consumer satisfaction and continuous use of AI [58]. Some researchers used the service robot acceptance model (sRAM) to show that contrary to popular belief, anthropomorphism does not always have a significant positive effect on voice assistants [39]. The flow theory has been used to show that the flow experience consumers perceive when interacting with voice assistants enhances their exploratory behaviour, hence their satisfaction and willingness to continue using voice assistants [44]. Social distance theory has shown that reducing social distances increases acceptance of verbally-interactive social robots [70], [71]. The uncanny valley theory [72] has been used to show that the morphology of robots affects consumers' attitudes towards the robot, which affects their adoption intention [25]. The computers are social actors (CASA) paradigm 
[73] was used to show that social attraction positively affects consumers' intentions to use robots [37]. Mind perception theory has been used to show that consumers intend to continue using voice assistants they perceive as competent and warm [42]. Lazarus's cognitionmotivation-emotion framework and social identity theory helped build an AI device use acceptance (AIDUA) model [3]. The model shows that emotions positively affect willingness to use AI devices in service delivery [3].

\subsection{Antecedents of AI adoption}

We identified 198 antecedents of AI adoption. Figure 3 presents a word cloud highlighting the most recurrent antecedents, while Figure 4 presents those that at least five studies have validated.

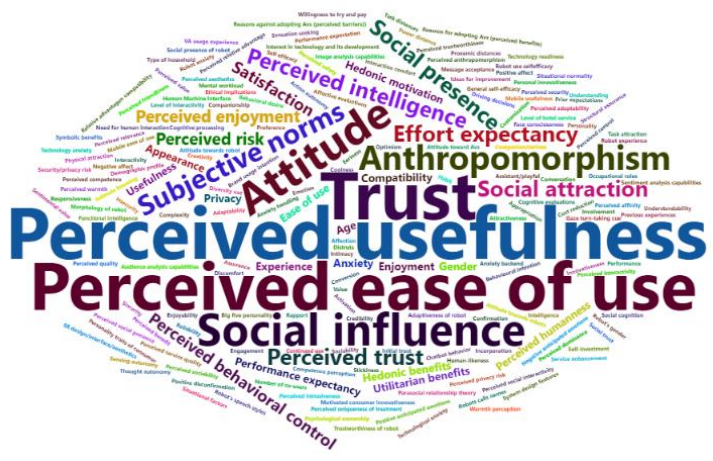

Figure 3. Antecedents of AI adoption by consumers

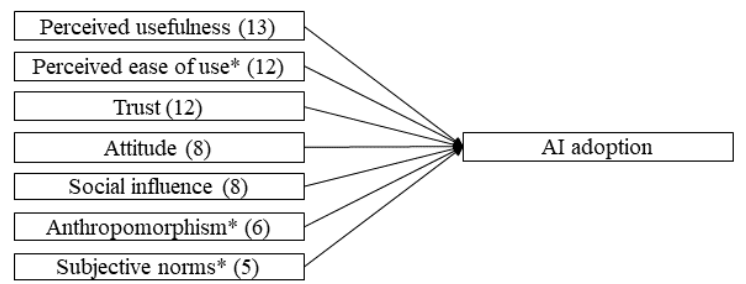

Figure 4. Most recurrent antecedents of AI adoption by consumers ${ }^{1 *}$

The antecedents of AI adoption presented in Figure 4 are also evidence of the main theories currently used to explain AI adoption by consumers. Perceived intelligence and perceived ease of use are antecedents highlighted by TAM. Attitude and subjective norms are mostly explained using the theory of planned behaviour. Meanwhile, social influence is mostly explained using UTAUT. Trust is highlighted as the most frequent antecedent of AI adoption by consumers after perceived usefulness and perceived ease of use. Theoretically, this antecedent has been explained using theories like service robot acceptance model [39], and by extending established theories like UTAUT [18], [32], [34], TAM [9], [46], [48], and parasocial relationship theory [40], [41]. Furthermore, anthropomorphism is the only antecedent of AI adoption that classic technology adoption theories do not explain.

Perceived usefulness has been defined as the extent to which a consumer believes using a particular system would improve their performance of a specific task [9]. It has also been defined as the ability of a robot to implement expected services [26]. Some researchers found that this variable has a direct positive effect on adoption intention [9], [39], [48], [52]. Meanwhile, it is affected by social influence [52]. Social influence also positively affects AI adoption intention [52]. However, some inconsistencies were identified in measurement scales. For example, measuring perceived usefulness by asking users if they think a robot adapts to their needs or if their friends have used robot services [26] is quite unconventional. Those items seem to measure other factors like adaptability and social influence.

Perceived ease of use has been defined as the extent to which a consumer believes that using a particular computer system is easy [9]. Another study describes it as the convenience of the robot and simplicity of operation [26]. Some researchers found that this variable has a direct positive effect on adoption intention in the context of service chatbots and autonomous vehicles [9], [48], [52]. However, perceived ease of use has been shown to have no significant effect on the intention to adopt voice assistants [39], [40]. Meanwhile, it has been shown to directly affect perceived usefulness [48], [52]. Perceived ease of use is also positively affected by social influence [52]. Some researchers measured perceived ease of use by asking consumers if they think the robot service looks and communicates like a natural person [26]. It seems the researchers were trying to measure anthropomorphism, thus creating confusion in the extant literature.

Trust has been defined as the psychological state of accepting to be vulnerable to another entity based on the expectation that the entity would not exploit that vulnerability negatively [49]. It has also been defined as the extent to which consumers perceive robots as reliable and credible [9]. Some researchers found that this variable has a direct positive effect on adoption intention [9], [28], [39], [48], [52], [63], [64]. Others found that trust affects perceived usefulness and perceived ease of use, affecting behavioural intention [34], [49]. Trust is also positively affected by openness

\footnotetext{
* Antecedents that sometimes did not affect AI adoption by consumers.
}

\footnotetext{
${ }^{1}$ Frequency in parentheses.
} 
and social influence and negatively affected by neuroticism personality traits [52].

Attitude has been defined as a consumer's assessment of a given behaviour [7]. It has also been defined as consumers' satisfaction with an AI's service [26]. Attitude has been found to positively affect AI adoption behaviour [7], [8], [63]. In addition, attitude is influenced by perceived usefulness and perceived ease of use [7]. Subjective norms positively affect AI adoption behaviour but not in all circumstances [7]. For example, it has been shown not to affect the acceptance of voice assistants [39].

Anthropomorphism has been defined as the extent to which consumers perceive robots as humanlike [9]. Some researchers found that this variable has a positive effect on adoption intention [9]. The strength of the impact depends on the clarity of communication during interaction and an individual's need for human interaction [54]. However, another study shows that anthropomorphism (perceived humanness) does not positively affect AI adoption [39]. Furthermore, there are conflicting results regarding this variable with respect to trust. Some studies find that anthropomorphism affects trust in humanoid robots, whereas others show no significant effect on trust in AI [40].

\section{Implications}

Like all technologies, consumers' AI adoption is essential for its existence and success [39], [49]. These findings have several implications for research on consumer adoption of AI technologies.

First, researchers need to clarify their understanding and conceptualisations of AI adoption. Using terms like adoption and acceptance interchangeably may be confusing for researchers, especially when the terms are not explicitly defined in the publication. Clearly defining these terms could help readers better understand the concept's operationalisation and the chosen measurement scales.

Second, more research is needed on AI adoption in specific aspects of the digital economy, like ecommerce. This paper highlights several controversial results in the extant literature resulting from differences in the application domain and AI system. Therefore, researchers should focus and emphasise a specific application or use case rather than investigate AI adoption of voice assistants or service robots in general. For example, based on the extant research, the anthropomorphism construct may be necessary for consumer services that humans initially performed. However, anthropomorphism may not be required for personal use, like searching the web using a voice assistant.
Third, this paper highlights different theoretical perspectives that have been used to explain consumer adoption of AI. It highlights the extensive use of traditional technology adoption theories and models to explain consumer adoption of AI. The theories still dominate this research stream despite several calls to propose alternative theoretical views to AI adoption. This paper reveals many inconsistent findings and unconventional adaptations of well-established constructs and measurement items. For example, it shows several instances where factors like ease of use highlighted by traditional models were not significant in explaining AI adoption. Such findings are strong indications that traditional theoretical perspectives are insufficient in explaining consumer adoption of AI. It implies that as AI adoption continues growing among consumers, researchers must increase their efforts towards developing or identifying novel theoretical perspectives that explain consumer AI adoption.

These findings align with previous studies that argue that some core constructs of traditional technology adoption theories are irrelevant when investigating consumer adoption of AI since consumer focus is different [47], [74]. For example, in conventional technologies, consumers need to learn how to use the technology. In contrast, in AI technologies, consumers instead focus on how well the technology can deliver human-level expertise [15], [71], [74]. Furthermore, the traditional theories are not comprehensive enough as they do not consider the anthropomorphic or social dimensions of consumer AI systems [3], [65].

The findings of this paper also validate several calls for research on AI adoption and acceptance. In IS research, it supports the need for more theories that consider the specificities of AI technologies to get a better grasp regarding their adoption [11]. Researchers are urged to investigate factors like culture as this could play an essential role in AI adoption given the differences in social behaviour worldwide [10], [75]. Researchers should also investigate factors like transparency/explicability, given that knowing why the AI takes specific actions may improve consumer adoption intention [10]. Other theories and concepts must thus be mobilised and advanced to explain AI adoption. We invite researchers to integrate other variables emphasised in this review, such as anthropomorphism which is a relevant concept in the context of AI, as it was shown more specifically in marketing research [43], [76]-[79]. Finally, this paper reveals directions for further research by providing a big picture of the main concepts and theoretical frameworks previously employed in various disciplines to explain AI adoption. 


\section{Conclusions}

This paper aims to provide a more holistic view and reveal essential gaps in the extant research on consumer adoption of AI. This paper intends to challenge researchers interested in consumer adoption of $\mathrm{AI}$ in the digital economy. It provides an evidence-based basis for researchers to argue and propose new theoretical perspectives to better understand consumer adoption of AI-powered systems used in today's vibrant economy. Our paper highlights the most studied technologies, the most used theories, and the most frequently investigated antecedents of AI adoption by consumers. Highlighting limitations and confusing findings in the extant literature contributes to advancing knowledge on fundamental questions in this research stream. It also draws the attention of researchers to the importance of the type of AI service or platform investigated and the context of use. These two aspects have been shown in this research to affect the determinants of AI adoption by consumers significantly. We hope this paper triggers the curiosity of researchers on consumer adoption of AI.

\section{References}

[1] A. Kaplan and M. Haenlein, "Siri, Siri, in my hand: Who's the fairest in the land? On the interpretations, illustrations, and implications of artificial intelligence," Bus. Horiz., vol. 62, no. 1, pp. 15-25, 2019.

[2] P. Mikalef and M. Gupta, "Artificial Intelligence Capability: Conceptualization, measurement calibration, and empirical study on its impact on organizational creativity and firm performance," Inf. Manag., p. 103434, 2021.

[3] D. Gursoy, O. H. Chi, L. Lu, and R. Nunkoo, "Consumers acceptance of artificially intelligent (AI) device use in service delivery," Int. J. Inf. Manage., vol. 49, pp. 157-169, 2019.

[4] P. M. Valkenburg and J. Cantor, "The development of a child into a consumer," J. Appl. Dev. Psychol., vol. 22, no. 1, pp. 61-72, 2001.

[5] M. R. Solomon, K. White, D. W. Dahl, J. L. Zaichkowsky, and R. Polegato, Consumer behavior: Buying, having, and being. Pearson Boston, MA, 2017.

[6] G. Atwal and D. Bryson, "Antecedents of intention to adopt artificial intelligence services by consumers in personal financial investing," Strateg. Chang., vol. 30, no. 3, pp. 293-298, May 2021.

[7] J. Pan, S. Ding, D. Wu, S. Yang, and J. Yang, "Exploring behavioural intentions toward smart healthcare services among medical practitioners: a technology transfer perspective.," Int. J. Prod. Res., vol. 57, no. 18, pp. 5801-5820, Sep. 2019.

[8] Y. Huang and L. Qian, "Understanding the potential adoption of autonomous vehicles in China: The perspective of behavioral reasoning theory,"
Psychol. Mark., vol. 38, no. 4, pp. 669-690, 2021. R. Pillai and B. Sivathanu, "Adoption of AI-based chatbots for hospitality and tourism," Int. J. Contemp. Hosp. Manag., vol. 32, no. 10, pp. 31993226, Oct. 2020.

[10] Y. K. Dwivedi et al., "Artificial Intelligence (AI): Multidisciplinary perspectives on emerging challenges, opportunities, and agenda for research, practice and policy," Int. J. Inf. Manage., p. 101994, Aug. 2019.

[11] P. J. Ågerfalk, "Artificial intelligence as digital agency," Eur. J. Inf. Syst., vol. 29, no. 1, pp. 1-8, 2020.

[12] R. E. Bawack, S. F. Wamba, and K. Carillo, “A framework for understanding artificial intelligence research: insights from practice," J. Enterp. Inf. Manag., vol. ahead-of-p, no. ahead-of-print, Jan. 2021.

[13] S. Raisch and S. Krakowski, "Artificial Intelligence and Management: The Automation-Augmentation Paradox," Acad. Manag. Rev., vol. 46, no. 1, 2020.

[14] K. Sohn and O. Kwon, "Technology acceptance theories and factors influencing artificial Intelligence-based intelligent products," Telemat. Informatics, vol. 47, p. 101324, 2020.

[15] R. Bawack, S. F. Wamba, and K. D. A. Carillo, "From IT to AI Artifact: Implications for IS Research on AI Adoption and Use," in Proceedings of the Twenty-Third DIGIT Workshop, 2018, no. 6, pp. 1-12.

[16] A. Harzing, "Journal quality list," Research in International Management, 2020. [Online]. Available: https://harzing.com/resources/journalquality-list.

[17] J. Webster and R. T. Watson, "Analyzing the Past to Prepare for the Future: Writing a Literature Review.," $M I S Q$., vol. 26, no. 2, pp. xiii-xxiii, 2002.

[18] N. Adnan, S. Md Nordin, M. A. bin Bahruddin, and M. Ali, "How trust can drive forward the user acceptance to the technology? In-vehicle technology for autonomous vehicle," Transp. Res. Part A Policy Pract., vol. 118, pp. 819-836, 2018.

[19] D. Belanche, L. V. Casaló, and C. Flavián, "Frontline robots in tourism and hospitality: service enhancement or cost reduction?," Electron. Mark., 2020.

[20] S. Borau, T. Otterbring, S. Laporte, and S. Fosso Wamba, "The most human bot: Female gendering increases humanness perceptions of bots and acceptance of AI," Psychol. Mark., vol. 38, no. 7, pp. 1052-1068, 2021.

[21] A. H. Butt, H. Ahmad, M. A. S. Goraya, M. S. Akram, and M. N. Shafique, "Let's play: Me and my AI-powered avatar as one team," Psychol. Mark., 2021.

[22] A. Dillon and M. G. Morris, "User Acceptance of Information Technology: Theories and Models," Annu. Rev. Inf. Sci. Technol., vol. 31, pp. 3-32, 1996.

[23] A. Edelmann, S. Stümper, and T. Petzoldt, "Cross- 
cultural differences in the acceptance of decisions of automated vehicles," Appl. Ergon., vol. 92, p. 103346, 2021.

[24] R. Latikka, T. Turja, and A. Oksanen, "Self-efficacy and acceptance of robots," Comput. Human Behav., vol. 93, pp. 157-163, 2019.

[25] H. H. Shin and M. Jeong, "Guests' perceptions of robot concierge and their adoption intentions," Int. J. Contemp. Hosp. Manag., vol. 32, no. 8, pp. 26132633, Jan. 2020.

[26] L. Zhong, X. Zhang, J. Rong, H. K. Chan, J. Xiao, and H. Kong, "Construction and empirical research on acceptance model of service robots applied in hotel industry," Ind. Manag. Data Syst., vol. 121, no. 6, pp. 1325-1352, Jan. 2021.

[27] G. McCartney and A. McCartney, "Rise of the machines: towards a conceptual service-robot research framework for the hospitality and tourism industry," Int. J. Contemp. Hosp. Manag., vol. 13, no. 12, pp. 3835-3851, 2020.

[28] S. Park, "Multifaceted trust in tourism service robots," Ann. Tour. Res., vol. 81, Mar. 2020.

[29] D. Kim and S. Kim, "A model for user acceptance of robot journalism: Influence of positive disconfirmation and uncertainty avoidance," Technol. Forecast. Soc. Change, vol. 163, p. 120448, 2021.

[30] M. M. A. de Graaf, S. Ben Allouch, and T. Klamer, "Sharing a life with Harvey: Exploring the acceptance of and relationship-building with a social robot," Comput. Human Behav., vol. 43, pp. 1-14, 2015.

[31] M. S. Delgosha and N. Hajiheydari, "How human users engage with consumer robots? A dual model of psychological ownership and trust to explain post-adoption behaviours," Comput. Human Behav., vol. 117, p. 106660, 2021.

[32] M. Fridin and M. Belokopytov, "Acceptance of socially assistive humanoid robot by preschool and elementary school teachers," Comput. Human Behav., vol. 33, pp. 23-31, 2014.

[33] I. Gaudiello, E. Zibetti, S. Lefort, M. Chetouani, and S. Ivaldi, "Trust as indicator of robot functional and social acceptance. An experimental study on user conformation to iCub answers," Comput. Human Behav., vol. 61, pp. 633-655, 2016.

[34] J. Guggemos, S. Seufert, and S. Sonderegger, "Humanoid robots in higher education: Evaluating the acceptance of Pepper in the context of an academic writing course using the UTAUT," Br. $J$. Educ. Technol., vol. 51, no. 5, pp. 1864-1883, Sep. 2020.

[35] N. Piçarra and J.-C. Giger, "Predicting intention to work with social robots at anticipation stage: Assessing the role of behavioral desire and anticipated emotions," Comput. Human Behav., vol. 86, pp. 129-146, 2018.

[36] B. Tay, Y. Jung, and T. Park, "When stereotypes meet robots: The double-edge sword of robot gender and personality in human-robot interaction," Comput. Human Behav., vol. 38, pp. 75-84, 2014.
[37] S. S. Sundar, E. H. Jung, T. F. Waddell, and K. J. Kim, "Cheery companions or serious assistants? Role and demeanor congruity as predictors of robot attraction and use intentions among senior citizens," Int. J. Hum. Comput. Stud., vol. 97, pp. 88-97, 2017.

[38] S. Han and H. Yang, "Understanding adoption of intelligent personal assistants: A parasocial relationship perspective," Ind. Manag. Data Syst., vol. 118, no. 3, pp. 618-636, 2018.

[39] T. Fernandes and E. Oliveira, "Understanding consumers' acceptance of automated technologies in service encounters: Drivers of digital voice assistants adoption," J. Bus. Res., vol. 122, pp. 180191, 2021.

[40] S. Moussawi, M. Koufaris, and R. Benbunan-Fich, "How perceptions of intelligence and anthropomorphism affect adoption of personal intelligent agents," Electron. Mark., 2020.

[41] V. Pitardi and H. R. Marriott, "Alexa, she's not human but... Unveiling the drivers of consumers' trust in voice-based artificial intelligence," Psychol. Mark., vol. 38, no. 4, pp. 626-642, Apr. 2021.

[42] Q. Hu, Y. Lu, Z. Pan, Y. Gong, and Z. Yang, "Can AI artifacts influence human cognition? The effects of artificial autonomy in intelligent personal assistants," Int. J. Inf. Manage., vol. 56, 2021.

[43] E. Moriuchi, "An empirical study on anthropomorphism and engagement with disembodied AIs and consumers' re-use behavior," Psychol. Mark., vol. 38, no. 1, pp. 21-42, Jan. 2021.

[44] A. Poushneh, "Humanizing voice assistant: The impact of voice assistant personality on consumers' attitudes and behaviors," J. Retail. Consum. Serv., vol. 58, p. 102283, 2021.

[45] C.-W. (Chloe) Ki, E. Cho, and J.-E. Lee, "Can an intelligent personal assistant (IPA) be your friend? Para-friendship development mechanism between IPAs and their users," Comput. Human Behav., vol. 111, p. 106412, 2020.

[46] G. McLean, K. Osei-Frimpong, and J. Barhorst, "Alexa, do voice assistants influence consumer brand engagement? - Examining the role of AI powered voice assistants in influencing consumer brand engagement," J. Bus. Res., vol. 124, pp. 312328, Jan. 2021.

[47] P. Liu, Z. Xu, and X. Zhao, "Road tests of selfdriving vehicles: Affective and cognitive pathways in acceptance formation," Transp. Res. Part A Policy Pract., vol. 124, pp. 354-369, 2019.

[48] I. Panagiotopoulos and G. Dimitrakopoulos, "An empirical investigation on consumers' intentions towards autonomous driving," Transp. Res. Part C Emerg. Technol., vol. 95, pp. 773-784, 2018.

[49] Z. Xu, K. Zhang, H. Min, Z. Wang, X. Zhao, and P. Liu, "What drives people to accept automated vehicles? Findings from a field experiment," Transp. Res. Part C Emerg. Technol., vol. 95, pp. 320-334, 2018.

[50] R. H. Y. Ma, A. Morris, P. Herriotts, and S. Birrell, "Investigating what level of visual information 
inspires trust in a user of a highly automated vehicle," Appl. Ergon., vol. 90, p. 103272, 2021.

[51] N. Du et al., "Look who's talking now: Implications of AV's explanations on driver's trust, AV preference, anxiety and mental workload," Transp. Res. Part C Emerg. Technol., vol. 104, pp. 428-442, 2019.

[52] T. Zhang et al., "Automated vehicle acceptance in China: Social influence and initial trust are key determinants," Transp. Res. Part C Emerg. Technol., vol. 112, pp. 220-233, 2020.

[53] L. Li, K. Y. Lee, E. Emokpae, and S.-B. Yang, "What makes you continuously use chatbot services? Evidence from chinese online travel agencies," Electron. Mark., 2021.

[54] B. Sheehan, H. S. Jin, and U. Gottlieb, "Customer service chatbots: Anthropomorphism and adoption," J. Bus. Res., vol. 115, pp. 14-24, 2020.

[55] E. Van den Broeck, B. Zarouali, and K. Poels, "Chatbot advertising effectiveness: When does the message get through?," Comput. Human Behav., vol. 98, pp. 150-157, 2019.

[56] A. Capatina, M. Kachour, J. Lichy, A. Micu, A.-E. Micu, and F. Codignola, "Matching the future capabilities of an artificial intelligence-based software for social media marketing with potential users' expectations," Technol. Forecast. Soc. Change, vol. 151, p. 119794, 2020.

[57] S. Tucker, "E-commerce standard user interface: an E-menu system," Ind. Manag. Data Syst., vol. 108, no. 8, pp. 1009-1028, Jan. 2008.

[58] K. Lee, K. Y. Lee, and L. Sheehan, "Hey Alexa! A Magic Spell of Social Glue?: Sharing a Smart Voice Assistant Speaker and Its Impact on Users' Perception of Group Harmony," Inf. Syst. Front., vol. 22, no. 3, pp. 563-583, 2020.

[59] F. D. Davis, "Perceived usefulness, perceived ease of use, and user acceptance of information technology," MIS Q. Manag. Inf. Syst., vol. 13, no. 3, pp. 319-339, 1989.

[60] V. Venkatesh, M. G. Morris, G. B. Davis, and F. D. Davis, "User acceptance of information technology: Toward a unified view," MIS Q. Manag. Inf. Syst., vol. 27, no. 3, pp. 425-478, 2003.

[61] V. Venkatesh, J. Y. L. Thong, and X. Xu, "Consumer acceptance and use of information technology: Extending the unified theory of acceptance and use of technology," MIS Q. Manag. Inf. Syst., vol. 36, no. 1, pp. 157-178, 2012.

[62] I. Ajzen, "The theory of planned behavior," Organ. Behav. Hum. Decis. Process., vol. 50, no. 2, pp. 179-211, 1991.

[63] S. S. Cha, "Customers' intention to use robotserviced restaurants in Korea: relationship of coolness and MCI factors," Int. J. Contemp. Hosp. Manag., vol. 32, no. 9, pp. 2947-2968, Jan. 2020.

[64] S. Wang, H. Yu, X. Hu, and J. Li, "Participant or spectator? Comprehending the willingness of faculty to use intelligent tutoring systems in the artificial intelligence era," Br. J. Educ. Technol., vol. 51, no.
5, pp. 1657-1673, Sep. 2020.

[65] G. McLean and K. Osei-Frimpong, "Hey Alexa ... examine the variables influencing the use of artificial intelligent in-home voice assistants," Comput. Human Behav., vol. 99, pp. 28-37, 2019.

[66] E. M. Rogers, Diffusion of Innovations, 5th edition. 2003.

[67] M. Blut, C. Wang, N. V. Wünderlich, and C. Brock, "Understanding anthropomorphism in service provision: a meta-analysis of physical robots, chatbots, and other AI," J. Acad. Mark. Sci., 2021.

[68] M. M. E. van Pinxteren, R. W. H. Wetzels, J. Rüger, M. Pluymaekers, and M. Wetzels, "Trust in humanoid robots: implications for services marketing," J. Serv. Mark., vol. 33, no. 4, pp. 507518, 2019.

[69] D. Horton and R. Richard Wohl, "Mass communication and para-social interaction: Observations on intimacy at a distance," Psychiatry, vol. 19, no. 3, pp. 215-229, 1956.

[70] Y. Kim, S. S. Kwak, and M. Kim, “Am I acceptable to you? Effect of a robot's verbal language forms on people's social distance from robots," Comput.

Human Behav., vol. 29, no. 3, pp. 1091-1101, 2013.

[71] Y. Kim and B. Mutlu, "How social distance shapes human-robot interaction," Int. J. Hum. Comput. Stud., vol. 72, no. 12, pp. 783-795, 2014.

[72] M. Mori, "The Uncanny Valley (By Masahiro Mori (Translated by Karl F. MacDorman and Norri Kageki)," IEEE Robot. Autom. Mag., vol. 12, pp. 98-100, 2012.

[73] C. Nass, J. Steuer, and E. R. Tauber, "Computer are social actors," in Conference on Human Factors in Computing Systems - Proceedings, 1994, pp. 72-78.

[74] L. Lu, R. Cai, and D. Gursoy, "Developing and validating a service robot integration willingness scale," Int. J. Hosp. Manag., vol. 80, pp. 36-51, 2019.

[75] Y. Duan, J. S. Edwards, and Y. K. Dwivedi, "Artificial intelligence for decision making in the era of Big Data - evolution, challenges and research agenda," Int. J. Inf. Manage., vol. 48, pp. 63-71, 2019.

[76] X. Li and Y. Sung, "Anthropomorphism brings us closer: The mediating role of psychological distance in User-AI assistant interactions," Comput. Human Behav., vol. 118, p. 106680, 2021.

[77] G. Z. Karimova and V. P. Goby, "The adaptation of anthropomorphism and archetypes for marketing artificial intelligence," J. Consum. Mark., vol. 38, no. 2, pp. 229-238, Jan. 2021.

[78] S. Verma, R. Sharma, S. Deb, and D. Maitra, "Artificial intelligence in marketing: Systematic review and future research direction," Int. J. Inf. Manag. Data Insights, vol. 1, no. 1, p. 100002, 2021.

[79] A. Guha et al., "How artificial intelligence will affect the future of retailing," J. Retail., vol. 97, no. 1, pp. 28-41, 2021. 